\title{
ACCELERATION OF WEB CONTENT AND PAGE STRUCTURE MANAGEMENT USING SHORTCODE
}

Miloš Dobrojević

Sinergija University,

Bijeljina, Bosnia and Herzegovina

Correspondence:

Miloš Dobrojević

e-mail:

mdobrojevic@sinergija.edu.ba

\begin{abstract}
:
Web content management software (WCM software), delivering relevant customer experience, is of critical importance for modern business. Regardless of industry or company size, adequate content management strategy can improve sales, work efficiency, data security or team collaboration, each of these being important factors of running a successful business. Intuitive web content management will streamline daily work activities, thus enabling employees to focus on assigned tasks rather than spending work hours on content and documents management. Besides few popular and well established solutions, new software solutions are emerging on the market, providing even more efficient methods for online content management. Among them is Magma CMS which uses an alternative approach based on intensive shortcode usage in order to simplify the processes of web content development and page structure control.
\end{abstract}

Keywords:

Dynamic Web Content, Shortcode, Content Management System, Magma CMS, Web page structure.

\section{INTRODUCTION}

A web content management system (WCMS) is a computer application that supports the creation and modification of content on the world wide web. It supports multiple users in a collaborative environment [1], typically providing structured content management features such as the ability to store articles, documents, images, video and any other online content type [2]. Such software applications are used for websites and web portals that frequently create and update content, usually have different content sections, multiple editors or developed community, including newspapers or government web portals.

Regardless of WCMS type or specialization, web content management systems typically provide a basic set of common, yet mandatory features such as:

- MVC (model-view-controller) architecture

- Modularity and extensibility

- SEO-friendly URLs

- Users / groups management

- Templating engine

- WYSIWYG text editor 
The goal is to reduce the need to code from scratch, to provide unified user experience (UX) throughout the application, enable access permissions management for different sections and content types.

Although embedding of new functionalities is possible through the system of modules built by 3 rd party companies or individuals, in certain cases this may bring up the questions of system stability, quality and security.

Finally, in an attempt to increase the universality of the WCM software, system architects are embedding tools for management of content types, web forms or DB data extraction, which additionally increases the complexity of already complex web content management systems. This results in increased demand for specialized developers, prolonged training for content authors, and eventually increased budgets for development and implementation.

In order to simplify the processes of web content development and page structure management, an alternative approach based on intensive shortcode usage may arise as an optimal solution.

\section{WEB CONTENT MANAGEMENT}

WCMS must facilitate the process of web content creation and publication, without knowledge of programming or markup languages. Popular features are:

- WYSIWYG editor with preview feature

- Import or embedding capabilities for multimedia and other documents (images, video, audio, pdf etc.)

- Content search with full-text search capability.

For easier content browsing, WCMS should be capable of content delivery by categories, site sections or adequate keywords. International projects should provide content in different languages.

On news, wiki or e-commerce sites, the WCMS should provide possibility of content promotion by:

- Interlinking articles

- Cross linking articles and content exchange within multisite network

- Related or promoted articles (i.e. cross selling)

In term of animating customers and stimulating them to take part in website operation, functionalities such as reader feedback channels (e.g. commenting and rating articles, or forum section) and social network plugins may provide handy.

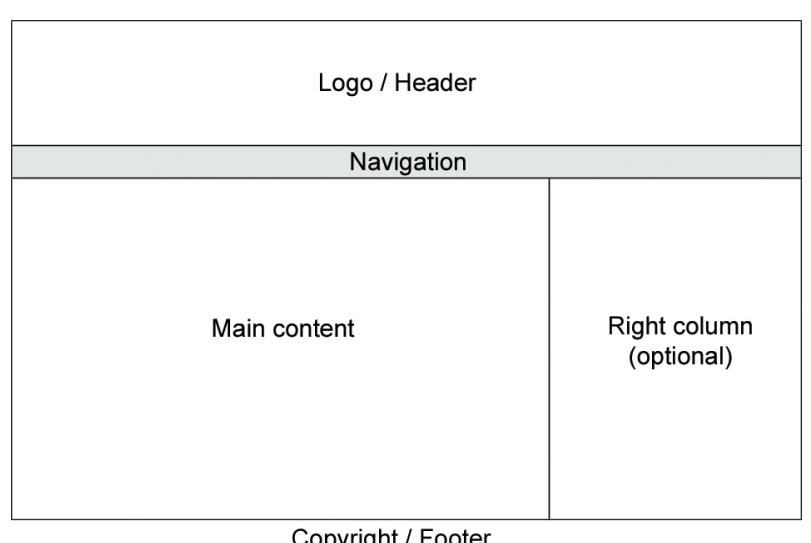

Copyright / Footer

Fig. 1. Simplified web page structure

\section{Web page structure}

Over the past two decades, the web has matured, and the structure of web pages based on text information become uniform and predictable. Although not all web pages share the exact layout and features, most of them incorporate basic components in standard page locations that have become familiar to users [3].

A dynamic web page may display different content each time it's viewed, i.e. depending on content update frequency, time of day, user's credentials etc. In case of a server-side dynamic web page, page generation process is controlled by a web application.

\section{Standard page layout elements}

Page structure implies standard mandatory elements, such as header, navigation panel, main content area and footer, figure 1 [4]. Optional elements such as user login, registration links, account panel, search form, content filters, slider, side column(s), SEO and social network widgets etc. may or may not be displayed, depending on WCMS capabilities, user's credentials or website usage scenario.

\section{Homepage design}

Homepage should be designed in such a manner to provide easy and effective navigation and usage of website or web application. In case of web applications, it should place visitors' attention to the most important information at the moment, while in the case of websites and web portals the role of homepage is to draw visitors' attention to products, services or information currently being in focus. 


\section{Content lists}

The primary goal of the Content List feature is to display content in a tabular format and provide user with the ability to quickly browse, navigate, and manage multiple content items. This is a dynamically generated list which can be adjusted depending on site configuration, user permissions and the individual content items.

\section{VESTI}

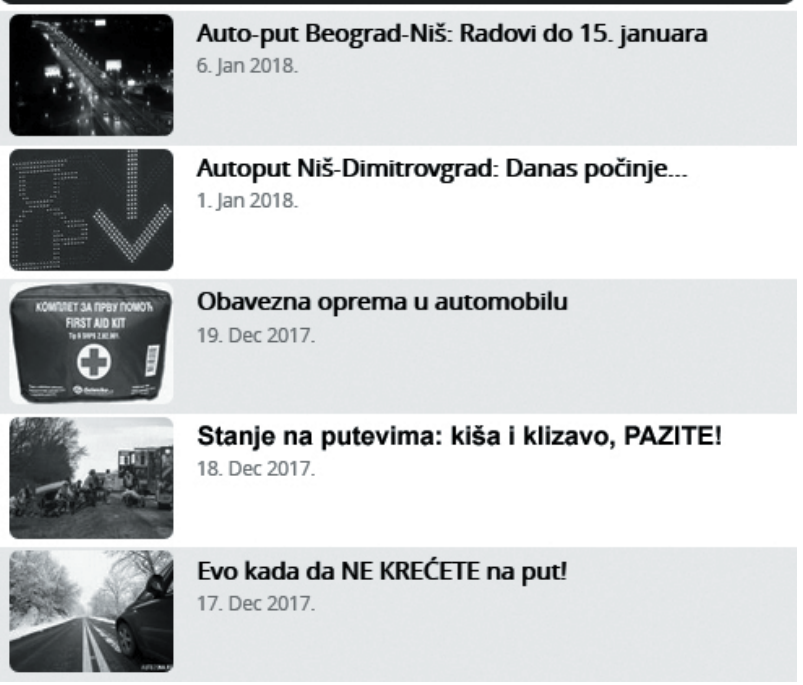

Fig. 2. Content list: the latest news, automotive web portal

\begin{tabular}{|c|c|c|}
\hline Popravljano & Cena & Početak / Kraj \\
\hline \. Tekuće održavanje & 0,00 & 01.12 .2015 \\
\hline$\bigwedge$ Gume & 0,00 & 30.06 .2015 \\
\hline A. Motot & 0,00 & 01.03 .2015 \\
\hline$\Lambda$ Zavarivanje izduvne grane ispred srednjeg lonca & 500,00 & 11.11 .2014 \\
\hline $\begin{array}{l}\text { Tekuće održavanje } \\
\text { Ulje Elf } 15 / 403 \times 11490 \mathrm{din} / 1\end{array}$ & $1.470,00$ & 21.08 .2014 \\
\hline
\end{tabular}

Fig. 3. Content list: current tasks, web based fleet management ERP system

Content list may contain article titles, task names, time info (date and time of publication, deadlines etc), depending on content type and usage scenario, i.e. news portal, classified ads website, web application or business information system. On figures 2 and 3 are shown respectively content lists on news web portal and web based ERP system (Magma Fleet Manager) [5], both powered by Magma CMS.

\section{LML, BBCODE, SHORTCODE}

Lightweight markup language (LML) represents a set of tags based on the HTML markup. They allow user to modify web content in the similar way as HTML does, but with a simpler syntax and without risk of breaking the web page layout $[6,7]$.

Historically, LML was originally used on displays incapable of showing neither graphics nor outline text (i.e. TrueType fonts, TTF). Such formatting was carried on to plain-text email communications, as well as console browsers.

In 1986 international standard SGML provided facilities to define and parse lightweight markup languages using grammars and tag implication [8].

\begin{tabular}{|c|c|c|}
\hline HTML/CSS & $\begin{array}{c}\text { Shortcode / } \\
\text { BBcode }\end{array}$ & Output \\
\hline $\begin{array}{l}<\mathrm{b}>\text { Text }</ \text { b }>\text { or } \\
<\text { strong }>\text { Text }</ \text { strong }>\text { or } \\
<\text { span style="font-weight: } \\
\text { bold" }>\text { Text }</ \text { span }>\end{array}$ & {$[\mathrm{b}] \mathrm{Text}[/ \mathrm{b}]$} & Text \\
\hline $\begin{array}{l}<\mathrm{i}>\text { Text }</ \mathrm{i}>\text { or } \\
<\text { em }>\text { Text }</ \text { em }>\text { or } \\
<\text { span style="font-style: } \\
\text { italic" }>\text { Text }</ \text { span }>\end{array}$ & {$[\mathrm{i}] \operatorname{Text}[/ \mathrm{i}]$} & Text \\
\hline$\langle\mathrm{b}\rangle\langle\mathrm{i}\rangle$ Text $\langle/ \mathrm{i}\rangle\langle/ \mathrm{b}\rangle$ & {$[\mathrm{b}][\mathrm{i}] \operatorname{Text}[/ \mathrm{i}][/ \mathrm{b}]$} & Text \\
\hline
\end{tabular}

Table 1. Simple text formatting by use of shortcode

The LML was widely used in the late 1990s and early 2000s to format posts in messaging boards (forum) software such as phpBB [9] or vBulletin [10], thus becoming known as the Bulletin Board Code, or BBCode.

Besides simple text formatting such as font weight, style, size or color (Table I), BBCode started to be used also for content aligning, embedding images and videos, posting links, listing items, tables etc.

In the last decade, major content management systems, e.g. Drupal, WordPress and Joomla embraced shortcode, through the system of 3rd party extensions or addons, as a tool which provides possibility of achieving complex, mostly visual effects by use of simple tags.

\section{Magma CMS $S^{\text {mix }}$ shortcode engine}

Unlike popular and well established WCMSs, Magma $\mathrm{CMS}^{\mathrm{mm}}$ possesses an advanced shortcode parsing engine already built in into system's core. In order to maximize security, use of shortcode tags is limited per user level [11]. 
Besides basic shortcode functionalities which are widely accepted among different CMSs, Magma $\mathrm{CMS}^{\mathrm{m}}$ also allows administrators to create page structure blocks such as main menu with submenus, slider, header or footer, to control page layout, content, or even entire site behavior just by use of simple tags, table II.

Furthermore, by combining different shortcode tags administrators and content editors are able to crosslink related articles, make reports and cross sections on submitted content, or insert commons that usually need to be coded (e.g. child or sub pages), thus massively reducing time required both for website or web application development, as well as content creation and management.

\section{DYNAMIC HOMEPAGE DESIGN WITH SHORTCODE}

\section{Page structure and content mapping}

As a use-case scenario, creation process of the home page of Auto Zona automotive web portal (www.autozona.rs, Fig. 4) can be analyzed. Page structure was mapped into sections, according to content nature they contain, Fig. 5. Three different types can be identified on the map:

\section{Functionality}

\section{Magma CMS shortcode}

\begin{tabular}{lc}
\hline Link internal article & {$[[$ Module code,Article ID, Link text $]]$} \\
\hline & {$[$ columns $]$} \\
Responsive content columns & Content in column \#1 \\
& {$[$ break $]$} \\
Content in column \#2 & {$[/$ columns $]$} \\
\hline
\end{tabular}

[plugin $=$

Block title,

Module code,

Internal content listing

Section IDs,

Number of articles and sort order,

Block widht,

Block alignment

]

[anonymous_content]

Content filtering based on the user level

Content visible only to

anonymous visitors

[/anonymous_content]

[menu]

[list]

[*][[Home $]]$

$\left[{ }^{*}\right][[\mathrm{News}]]$

$\left[{ }^{*}\right] \ldots$

$\left[{ }^{*}\right][[$ Contact $]]$

[/list]

[/menu]

Table 2. Basic Magma CMS’ shortcode functionalities 
- Static content. Once generated, it won't be further changed during longer periods of time. This type of content can be generated with HTML only, or by combination of HTML and available shortcode tags.

- Dynamic content is drawn from 'Articles' module, which on the other hand contains two zones. This requires use of [plugin] shortcode tags.

»Zone 1 contains news articles related to automotive industry.

»Zone 2 contains entertainment articles related to cars, trucks and traffic.

- Dynamic external content is retrieved from other web sites within the same Magma CMS multisite network. This can be accomplished by use of the [widget] shortcode tag.

\section{Content lists}

Content list (Figure 2, homepage slot shown on Figures 4 and 5) will be generated by Magma CMS shortcode parser (Shortcode 1 [12]), containing the latest five articles from the zone 1 of the module "Articles".

The last two parameters are optional, representing list width and alignment. This process is automatic and does not require any further action from the administrators. However, content list can be additionally stylized with the CSS code, embedded either into the article body or included as a separate stylesheet.

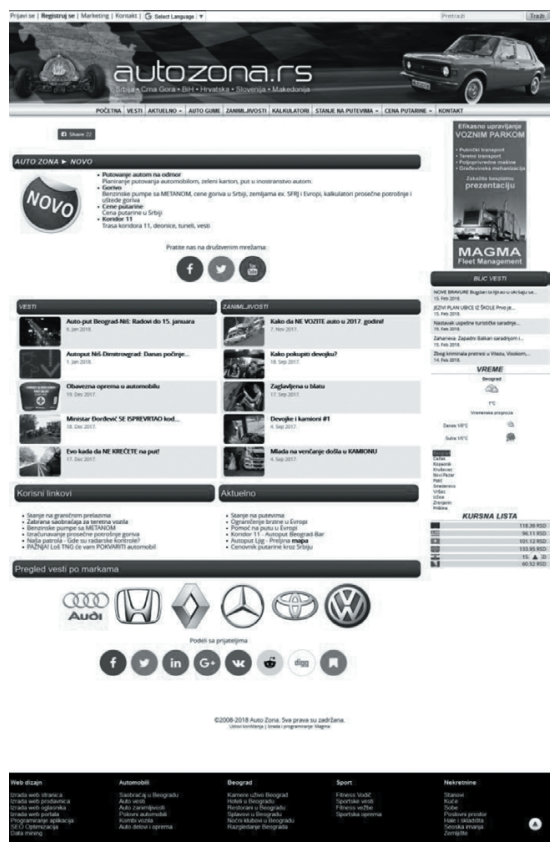

Fig. 4. Web portal powered by Magma CMS, homepage with embedded content lists
Shortcode. 1. Magma CMS - embedding content list with a simple shortcode (BBcode)

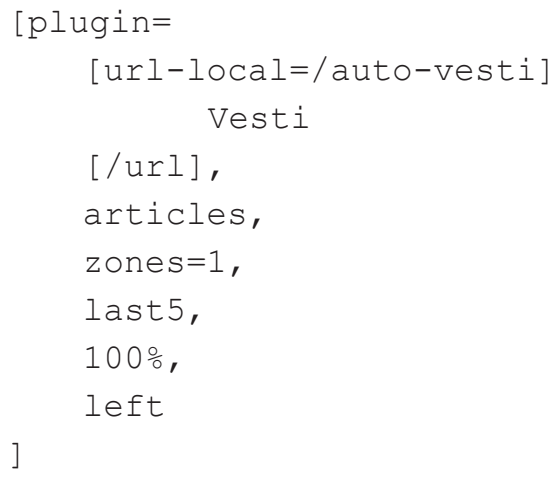

Banners

In Magma $\mathrm{CMS}^{\text {ma }}$ terminology, the term "banner" implies much broader usage scenario other than placing ads. Banners can be comprehended as web page building elements, similar to Drupal's structure blocks. Besides pictures, banners can also contain HTML, CSS and JavaScript code, as well as shortcode tags, thus making them an effective tool for dynamic page layout control without need to modify the site template.

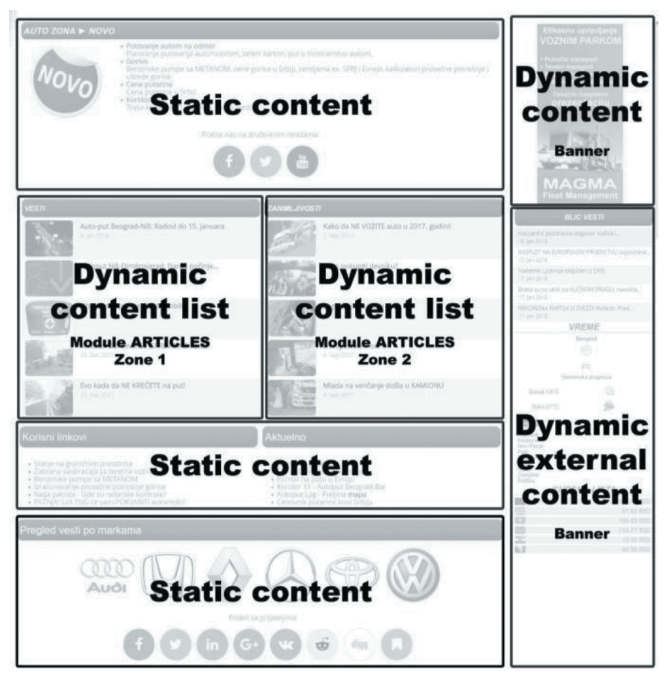

Fig. 5. Home page layout map by content type

Vice versa, although banners management and behavior is primarily controlled within the banners module, it also can be controlled by use of appropriate shortcode tags from both the article body and banners.

Shortcode tag [no_banners] can disable one or more banner slots in the web page layout. In the example shown in shortcode 2 [12], [no_banners] tag is used in the article body to remove right column from the page layout. 
Shortcode. 2. Magma CMS - control page

layout with shortcode

[no_banners]right_column [/no_banners]

\section{Home page coding}

Finally, workflow of home page (figures 4 and 5) development with Magma $\mathrm{CMS}^{\text {min }}$ can be done in a single step by creating a home page article, provided that the user is already logged in with proper credentials.

Besides minor HTML usage, the whole page content and layout control are handled with proper shortcode tags built in within the Magma $\mathrm{CMS}^{\text {mix }}$ core. Shortcode snippet in charge for dynamic content lists (Figure 5) is shown in Shortcode 3.

Shortcode. 3. Magma CMS - dynamic content lists

[columns] [plugin=[url-local=/auto-vesti]Vesti [/url], articles, zones=1, last5, $100 \%$, left]

[break] [plugin=[url-local=/auto-zanimljivosti] Zanimljivosti [/url], articles, zones=2, last5, 100\%] [/columns ]

Instead of placing content lists into HTML table, [columns] shortcode tag was used which in turn automatically generates responsive layout: on desktop view content lists will be displayed horizontally, and on mobile devices they will be laid down one beneath the other.

Once created, page body code can be left unchanged for long periods of time. Page content will be automatically updated by software, providing dynamic information on the latest news and articles to site visitors.

\section{CONCLUSION}

Although well established WCMS solutions such as WordPress, Drupal or Joomla are omnipresent and accepted as industry standard, smaller and not so well known content management systems can prove to be more optimal and more efficient tools in terms of workflow optimization and reduction of working hours required for certain tasks.

Majority of CMS software available on the market tend to comply certain architecture and set of features, making difficult for a regular user to analyze and choose optimal solution.

The tendency of incorporating database management tools into CMS software in terms of getting an universal tool for content management, combined with overly segmentation through numerous modules, plugins or extensions induced by a specific business model in the open source industry, inevitable leads to an oxymoron: software which was initially produced to provide content management capability for users without programming knowledge, eventually transforms into complex, cumbersome solution which demands experienced, pricey developers to perform simple tasks.

In this paper, on the simple use case example, was demonstrated an alternative approach on how certain scenarios in web development can be solved in a simple, yet very efficient manner.

LML, shortcode or BBcode tags incorporated into Magma CMS' core proved to be a tool capable of significantly reducing the need for additional coding, modules development as well as time required for

- control of web page structural elements without need for additional modules or template modification

- dynamic content embedment

- fetching and mixing of content originated from different site sections into a single content list

- content distribution based on user level or credentials

- content cross linking

- content exchange in multisite networks

This is achieved by

- reduction of steps (and thus work hours) required for the task completion, to a bare minimum.

- eliminating the need to develop additional backend (PHP) code or templates.

On the other hand, the required knowledge level is kept on an acceptable level, so that less experienced developers can be engaged in completing such tasks, without the risk of breaking either the application or the budget.

The final result is increased productivity with reduced risk factor, while production costs remain the same or get reduced as well.

\section{REFERENCES}

[1] Karl Paulsen, Focal Press (2011) Moving Media Storage Technologies: Applications \& Workflows for Video and Media, 381.

[2] BuiltWith, CMS Usage Statistics: Statistics for websites using CMS technologies, retrieved from https://trends.builtwith.com/cms 
[3] P.J. Lynch, S. Horton, Page Structure and Site Design, Web Style Guide, retrieved from http:// webstyleguide.com/wsg3/6-page-structure/3-sitedesign.html

[4] M. Dobrojevic, "Kako napraviti web aplikaciju", Magma.rs, 2016, ISBN 978-86-920027-0-0, p26

[5] M. Dobrojević, "Važnost primene softvera za upravljanje resursima i upotrebe 'data mining' tehnika za prevenciju zloupotrebe u flotnom menadžmentu”, 2017, Bezbednost, Beograd, vol. 59, no. 3, pp. 79100.

[6] Wikipedia, Lightweight markup language, retrieved from https://en.wikipedia.org/wiki/Lightweight_ markup_language

[7] Ubuntu forums, BB Code List, retrieved from https://ubuntuforums.org/misc.php?do=bbcode
[8] International Organization for Standardization,ISO 8879:1986, Information processing - Text and office systems - Standard Generalized Markup Language (SGML), retrieved from https://www.iso.org/standard/16387.html

[9] phpBB,BBCode guide, retrieved from https://www. phpbb.com/community/help/bbcode

[10] vBulletin, BB Code List, retrieved from https:// www.vbulletin.org/forum/misc.php?do=bbcode

[11] Magma, BBCode, retrieved from http://magmacms. org/features/bbcode

[12] M. Dobrojevic, “ Magma CMS: Enhancement of content management productivity", International Economics and Management Conference ECIN 2017, ISBN 978-86-7329-105-5 